\title{
Design, synthesis and antimicrobial evaluation of pyrimidin-2-ol/thiol/amine analogues
}

Sangeeta Narwal, Sanjiv Kumar and Prabhakar Kumar Verma*

\begin{abstract}
Background: Pyrimidine is an aromatic heterocyclic moiety containing nitrogen atom at 1st and 3rd positions and play an important role to forms the central core for different necessity of biological active compounds, from this facts, we have designed and synthesized a new class of pyrimidin-2-ol/thiol/amine derivatives and screened for its in vitro antimicrobial activity.

Results and discussion: The synthesized pyrimidine derivatives were confirmed by $I R,{ }^{1} \mathrm{H} /{ }^{13} \mathrm{C}-\mathrm{NMR}$, Mass spectral studies and evaluated for their in vitro antimicrobial potential against Gram positive (S. aureus and B. subtilis), Gram negative (E. coli, P. aeruginosa and S. enterica) bacterial strains and fungal strain (C. albicans and A. niger) by tube dilution method and recorded minimum inhibitory concentration in $\mu \mathrm{M} / \mathrm{ml}$. The MBC and MFC values represent the lowest concentration of compound that produces in the range of $96-98 \%$ end point reduction of the used test bacterial and fungal species.
\end{abstract}

Conclusion: In general all synthesized derivatives exhibited good antimicrobial activity. Among them, compounds $\mathbf{2}$, $\mathbf{5 , 1 0 , 1 1}$ and $\mathbf{1 2}$ have significant antimicrobial activity against used bacterial and fungal strains and also found to be more active than the standard drugs.

Keywords: Pyrimidine derivatives, Antibacterial activity, Antifungal activity

\section{Background}

Antimicrobial agents are one of the most important weapons in the resistance of infection caused by bacterial strains. In the past few years, increase the resistance of microorganisms toward antimicrobial agents become a serious health problem so there is a need of safe, potent and novel antimicrobial agents [1]. Pyrimidine aromatic heterocyclic moiety containing nitrogen atom at 1st and 3rd positions and play an important role to forms the central core for different necessity of biological active compounds [2]. Pyrimidine is the structural unit of DNA and RNA which play an imperative role in various existence progressions. Pyrimidines are present among the three isomeric diazines. Most abundant pyrimidine is uracil, cytosine and thymine [3]. These derivatives are

*Correspondence: vermapk422@rediffmail.com Department of Pharmaceutical Sciences, Maharshi Dayanand University, Rohtak, Haryana 124001, India also known as $m$-diazine or 1,3-diazone can be regarded as cyclic amine and shows the various biological activities i.e. antiviral [4, 5]; anticancer [6]; antimicrobial [7]; antiinflammatory [8]; analgesic [9]; antioxidant [10]; antimalarial [11].

Pyrimidine is used as parent substance for the synthesis of a wide variety of heterocyclic compounds and raw material for the synthesis of new molecule [12]. Pyrimidine ring complexes with different heterocyclic moiety found to be an essential part of natural products agrochemicals and veterinary products. A large measure of antimicrobial drugs such as ciprofloxacin, chloramphenicol, griseofulvin and nystatin are available for bacterial and fungal infections [13].

Recently, it was reported that $p$-methoxyphenyl group present on pyrimidine nucleus improved the antimicrobial activity of the pyrimidine derivative (I) [13], $p$-Chloro phenyl group present on pyrimidine nucleus 
[14] improved the anticancer activity of the pyrimidine derivatives (II), $p$-Methoxyphenyl group present on pyrimidine derivatives (III) improved the antioxidant [15], $p$-Methoxyphenyl group present on pyrimidine ring (IV) improved the antitubercular activity of the pyrimidine derivatives [16], $p$-Hydroxy group present on pyrimidine nucleus $(\mathbf{V})$ improved the antimicrobial of the pyrimidine compound [10]. The electron releasing ($\mathrm{OH}$ and $\left.-\mathrm{OCH}_{3}\right)$ and electron withdrawing $(-\mathrm{Cl})$ groups are present on different position of pyrimidine nucleus (I, II, III, IV and V) enhanced the biological activity of the pyrimidine derivatives, from this facts we developed a design of reported biological active agents and proposed antimicrobial agent which is presented in Fig. 1. In light of abovementioned facts, we hereby report to design, synthesis and antimicrobial screening of 4-(substituted phenyl)-6-(4-nitrophenyl) pyrimidin-2-ol/thiol/amine derivatives (Scheme 1a, b).

\section{Results and discussion Chemistry}

Synthesis of pyrimidine derivatives (1-13) followed the general procedure discussed in synthetic Scheme 1a, b. The reaction of substituted chalcone in the presence of guanidine hydrochloride/urea/thiourea in methanolic solvent resulted in the formation of the final compounds. The physicochemical properties of newly synthesized compounds are presented in Table 1. The molecular structures of the synthesized compounds (1-13) were confirmed by FT-IR (KBr pellets, $\mathrm{cm}^{-1}$ ) and ${ }^{1} \mathrm{H} /{ }^{13} \mathrm{C}$ NMR $\left(\mathrm{CDCl}_{3}, \delta \mathrm{ppm}\right)$ spectral and elemental studies. The appearance of IR absorption band at $1404 \mathrm{~cm}^{-1}$ in the spectral data of synthesized derivatives (1-13) displayed the presence of $\mathrm{Ar}-\mathrm{OH}(\mathrm{C}-\mathrm{O}$ str. and $\mathrm{O}-\mathrm{H}$ in plane bend. vib.) category on the aromatic ring. The IR absorption band in the scale of $645-623 \mathrm{~cm}^{-1}$ corresponds to the $\mathrm{C}-\mathrm{Br}$ stretching of aromatic-bromo compounds (10 and 11). The existence of $\mathrm{Ar}-\mathrm{NO}_{2}$ group asymmetric $\mathrm{Ar}-\mathrm{NO}_{2}$ stretches in the scale of $1550-1510 \mathrm{~cm}^{-1}$. The existence of an arylalkyl ether category $\left(\mathrm{Ar}-\mathrm{OCH}_{3}\right)$ in compounds 8 and 9 are established by the existence of an IR absorption band around $2842-2829 \mathrm{~cm}^{-1}$. Halogen group in compounds 1-7 and $\mathbf{1 2}$ is indicated by the existence of $\mathrm{Ar}-\mathrm{Cl}$ stretching vibrations at $732-848 \mathrm{~cm}^{-1}$. The impression of IR stretching at 2602-2627 and $623-709 \mathrm{~cm}^{-1}$ in the spectral data of synthesized compounds specified the existence of $\mathrm{S}-\mathrm{H}$ and $\mathrm{C}-\mathrm{S}$ group respectively. The appearance of IR stretching at 3379$3349 \mathrm{~cm}^{-1}$ spectral data of synthesized compounds specified the existence of $-\mathrm{NH}_{2}$ group. The impression of IR stretching vibration at $3100-3000$ and $1580-1600 \mathrm{~cm}^{-1}$ in the spectral data of synthesized compounds specified the existence of $\mathrm{C}-\mathrm{H}$ and $\mathrm{C}=\mathrm{C}$ group respectively.
The appearance of IR stretching $1670-1709 \mathrm{~cm}^{-1}$ in the spectral data of all synthesized compounds specified the existence of $\mathrm{C}=\mathrm{N}$ group. The multiplet signals between 6.33 and $8.34 \delta \mathrm{ppm}$ in ${ }^{1} \mathrm{H}$-NMR spectra is indicative of aromatic proton of synthesized derivatives. The compounds 8 and 9 showed singlet at 3.01-3.34 $\delta \mathrm{ppm}$ due to the existence of $\mathrm{OCH}_{3}$ of $\mathrm{Ar}-\mathrm{OCH}_{3}$. All compounds showed singlet at 7.51-8.43 and 6.85-841 $\delta \mathrm{ppm}$ due to the existence of $\mathrm{N}=\mathrm{CH}$ and $-\mathrm{CH}$ groups in pyrimidine ring respectively. Compound $\mathbf{1 3}$ showed singlet at $2.19 \delta \mathrm{ppm}$ due to existence of $-\mathrm{N}\left(\mathrm{CH}_{3}\right)_{2}$ at the para position. Compounds, $1,3,5,8,11$ and 12 showed singlet at 4.0-4.3 $\delta \mathrm{ppm}$ due to existence of $-\mathrm{NH}_{2}$ at the para position and 2, 4, 6 and $\mathbf{1 0}$ showed singlet at 3.01$3.34 \delta \mathrm{ppm}$ due to existence of $-\mathrm{SH}$ group at the para position of the pyrimidine ring. The elemental screened studies of the 4-(substituted phenyl)-6-(4-nitrophenyl) pyrimidin-2-ol/thiol/amine were found within $\pm 0.39 \%$ of the theoretical results.

\section{In vitro antimicrobial activity}

All the newly synthesized pyrimidine derivatives were examined for their in vitro antimicrobial activity against Gram positive S. aureus (MTCC 3160), B. subtilis (MTCC 441), Gram negative species: E. coli (MTCC 443), P. aeruginosa (MTCC 3542), S. enteric (MTCC 1165) and fungus species: A. niger (MTCC 281) and C. albicans (MTCC 227) strain using tube dilution method [17]. Dilutions of test and standard compounds were prepared in double strength nutrient broth for bacterial strains and sabouraud dextrose broth for fungal strains [18]. The minimum inhibitory concentration (MIC i.e. lowest concentration required of test substance to complete growth inhibition) values of standard drugs and synthesized compounds are presented in Table 2. From the results of antimicrobial evaluation it was observed that the entire synthesized compounds showed appreciable antimicrobial activity and different compounds were found to be active against different microorganisms. In case of Gram positive bacteria, compounds $12\left(\mathrm{MIC}_{\mathrm{sa}}=0.87 \mu \mathrm{M} /\right.$ $\mathrm{ml}$ ) showed significant activity against $S$. aureus and $\mathbf{5}$ $\left(\mathrm{MIC}_{\mathrm{bs}}=0.96 \mu \mathrm{M} / \mathrm{ml}\right)$ exhibited most potent antibacterial activity against $B$. subtilis. In case of Gram negative bacteria, compounds $10\left(\mathrm{MIC}_{\mathrm{se}}=1.55 \mu \mathrm{M} / \mathrm{ml}\right)$ showed significant activity against Salmonella enteric, 2 $\left(\mathrm{MIC}_{\mathrm{ec}}=0.91 \mu \mathrm{M} / \mathrm{ml}\right)$ displayed more potent antibacterial activity against E. coli and $\mathbf{1 0}\left(\mathrm{MIC}_{\mathrm{pa}}=0.77 \mu \mathrm{M} /\right.$ $\mathrm{ml}$ ) exhibited most potent antibacterial activity against P. aeruginosa. Compound $12\left(\mathrm{MIC}_{\mathrm{ca}}=1.73 \mu \mathrm{M} / \mathrm{ml}\right)$ showed significant activity against $C$. albicans and $\mathbf{1 1}$ $\left(\mathrm{MIC}_{\mathrm{an}}=1.68 \mu \mathrm{M} / \mathrm{ml}\right)$ was found to be most potent antifungal agent against $A$. niger. All synthesized compounds having more antimicrobial potential than the standard 


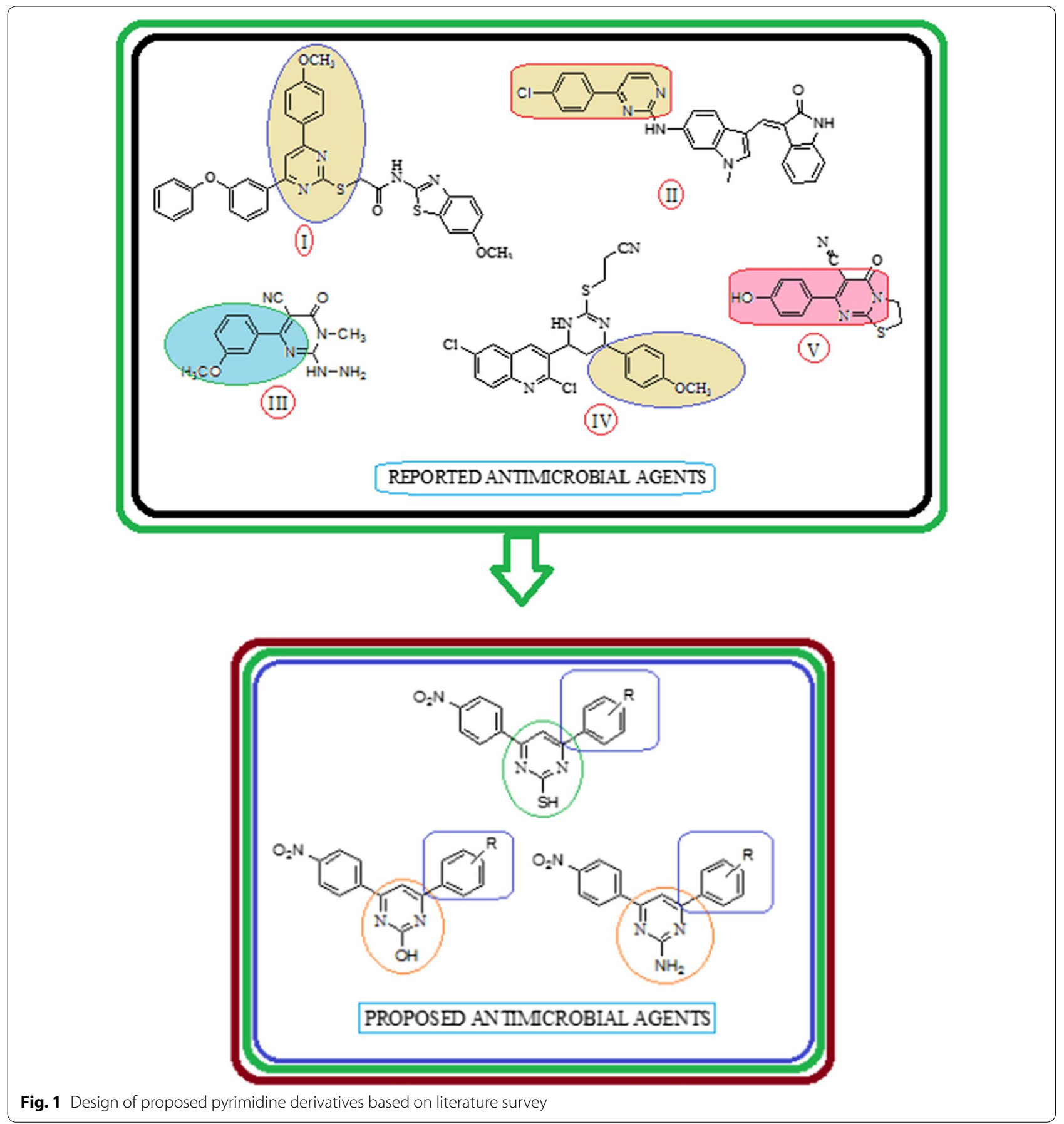

cefadroxil (antibacterial) and fluconazole (antifungal) drugs and these compounds may be used as lead for the further discovery of new antimicrobial agents.

\section{Determination of $M B C / M F C$}

After recorded the MIC results of the synthesized compounds in concentration of $(50,25,12.5,6.25,3.125$, 1.56) $\mu \mathrm{M} / \mathrm{ml}$ against microbial species i.e. Gram positive bacteria (S. aureus and B. subtilis), Gram negative bacteria ( $E$. coli, $P$. aeruginosa and $S$. enterica) and fungal strain ( $C$. albicans and A. niger) then their minimum bactericidal concentration (MBC) and fungicidal concentration (MFC) were determined by petri dish method using nutrient agar media (antibacterial) and sabouraud dextrose agar media (antifungal) by subculturing $100 \mu \mathrm{l}$ of culture from each test tube that remained clear in the 

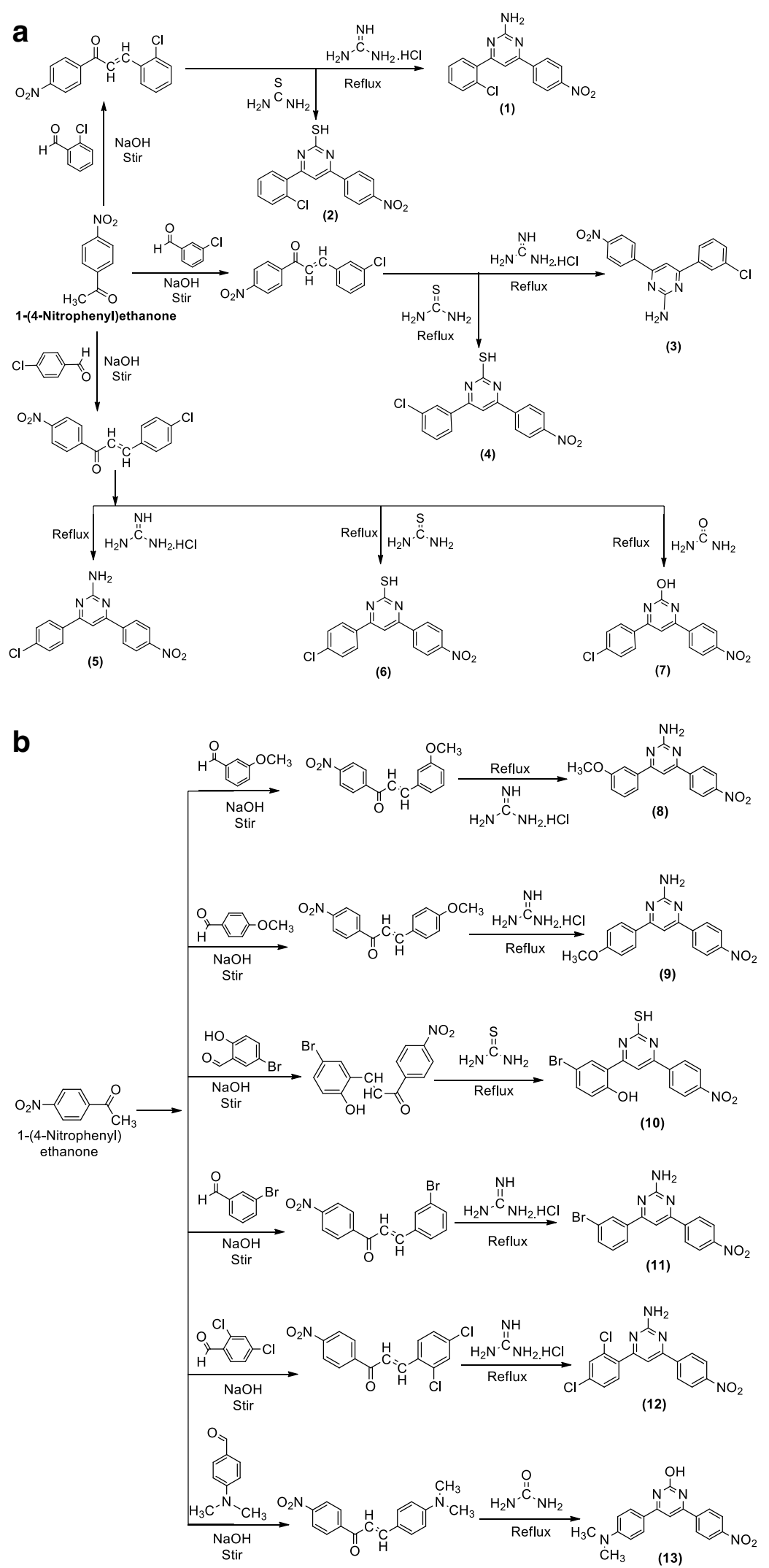

Scheme 1 a, b Synthesis of 4-(substituted phenyl)-6-(4-nitrophenyl)pyrimidin-2-ol/thiol/amine derivatives 
Table 1 The physicochemical properties of synthesized 4-(substituted phenyl)-6-(4-nitrophenyl) pyrimidin-2-ol/thiol/ amine derivatives

\begin{tabular}{|c|c|c|c|c|c|}
\hline Compounds & M. formula & M. weight & M.pt. $\left({ }^{\circ} \mathrm{C}\right)$ & $\mathrm{R}_{f}$ value $^{\mathrm{a}}$ & $\%$ Yield \\
\hline \multicolumn{6}{|c|}{ Physicochemical properties } \\
\hline 1. & $\mathrm{C}_{16} \mathrm{H}_{11} \mathrm{ClN}_{4} \mathrm{O}_{2}$ & 326 & $80-82$ & 0.45 & 75.00 \\
\hline 2. & $\mathrm{C}_{16} \mathrm{H}_{10} \mathrm{ClN}_{3} \mathrm{O}_{2} \mathrm{~S}$ & 343 & $61-63$ & 0.57 & 84.72 \\
\hline 3. & $\mathrm{C}_{16} \mathrm{H}_{11} \mathrm{ClN}_{4} \mathrm{O}_{2}$ & 326 & $76-78$ & 0.60 & 78.78 \\
\hline 4. & $\mathrm{C}_{16} \mathrm{H}_{10} \mathrm{ClN}_{3} \mathrm{O}_{2} \mathrm{~S}$ & 343 & $90-92$ & 0.62 & 72.54 \\
\hline 5. & $\mathrm{C}_{16} \mathrm{H}_{11} \mathrm{ClN}_{4} \mathrm{O}_{2}$ & 326 & $122-124$ & 0.58 & 82.22 \\
\hline 6. & $\mathrm{C}_{16} \mathrm{H}_{10} \mathrm{ClN}_{3} \mathrm{O}_{2} \mathrm{~S}$ & 343 & $63-65$ & 0.56 & 75.00 \\
\hline 7. & $\mathrm{C}_{16} \mathrm{H}_{10} \mathrm{ClN}_{3} \mathrm{O}_{3}$ & 327 & $127-129$ & 0.60 & 84.72 \\
\hline 8. & $\mathrm{C}_{17} \mathrm{H}_{14} \mathrm{~N}_{4} \mathrm{O}_{3}$ & 322 & $66-68$ & 0.51 & 73.43 \\
\hline 9. & $\mathrm{C}_{17} \mathrm{H}_{14} \mathrm{~N}_{4} \mathrm{O}_{3}$ & 322 & $89-91$ & 0.56 & 76.47 \\
\hline 10. & $\mathrm{C}_{16} \mathrm{H}_{10} \mathrm{BrN}_{3} \mathrm{O}_{3} \mathrm{~S}$ & 404 & $59-61$ & 0.61 & 81.81 \\
\hline 11. & $\mathrm{C}_{16} \mathrm{H}_{11} \mathrm{BrN}_{4} \mathrm{O}_{2}$ & 371 & $153-155$ & 0.41 & 64.00 \\
\hline 12. & $\mathrm{C}_{16} \mathrm{H}_{10} \mathrm{ClN}_{4} \mathrm{O}_{2}$ & 361 & $87-89$ & 0.42 & 87.61 \\
\hline 13. & $\mathrm{C}_{18} \mathrm{H}_{16} \mathrm{~N}_{4} \mathrm{O}_{3}$ & 336 & $156-158$ & 0.45 & 77.38 \\
\hline
\end{tabular}

a TLC mobile phase-benzene

Table 2 Antimicrobial activity (MIC $=\mu \mathrm{M} / \mathrm{ml}$ ) of synthesized 4-(substituted phenyl)-6-(4-nitrophenyl) pyrimidin-2-ol/ thiol/amine derivatives

\begin{tabular}{|c|c|c|c|c|c|c|c|}
\hline \multirow[t]{4}{*}{ Compounds no. } & \multicolumn{7}{|c|}{ Minimum inhibitory concentration $(\mathrm{MIC}=\mu \mathrm{M} / \mathrm{ml})$} \\
\hline & \multicolumn{5}{|c|}{ Bacterial strains } & \multirow{2}{*}{\multicolumn{2}{|c|}{ Fungal strains }} \\
\hline & \multicolumn{2}{|l|}{ Gram positive } & \multicolumn{3}{|c|}{ Gram negative } & & \\
\hline & $\begin{array}{l}\text { S. aureus } \\
\text { (MTCC } 3160 \text { ) }\end{array}$ & $\begin{array}{l}\text { B. subtilis } \\
\text { (MTCC 441) }\end{array}$ & $\begin{array}{l}\text { E. coli } \\
\text { (MTCC 443) }\end{array}$ & $\begin{array}{l}\text { P. aeruginosae } \\
\text { (MTCC 3542) }\end{array}$ & $\begin{array}{l}\text { S. enteric } \\
\text { (MTCC 1165) }\end{array}$ & $\begin{array}{l}\text { C. albicans } \\
\text { (MTCC 227) }\end{array}$ & $\begin{array}{l}\text { A. Niger (MTCC } \\
\text { 281) }\end{array}$ \\
\hline 1. & 1.91 & 3.83 & 1.91 & 1.91 & 1.91 & 3.83 & 3.83 \\
\hline 2. & 1.82 & 3.64 & 0.91 & 1.82 & 1.82 & 1.82 & 3.64 \\
\hline 3. & 1.91 & 3.83 & 0.96 & 1.91 & 3.83 & 3.83 & 3.83 \\
\hline 4. & 3.64 & 3.64 & 1.82 & 0.91 & 3.64 & 3.64 & 3.64 \\
\hline 5. & 1.91 & 0.96 & 1.91 & 1.91 & 3.83 & 1.91 & 3.83 \\
\hline 6. & 1.82 & 3.64 & 1.82 & 1.82 & 3.64 & 1.82 & 3.64 \\
\hline 7. & 3.81 & 3.81 & 1.91 & 1.91 & 3.81 & 1.91 & 3.81 \\
\hline 8. & 3.88 & 3.88 & 1.94 & 3.88 & 3.88 & 1.94 & 3.88 \\
\hline 9. & 1.94 & 3.88 & 1.94 & 3.88 & 3.88 & 3.88 & 3.88 \\
\hline 10. & 3.09 & 1.55 & 1.55 & 0.77 & 1.55 & 3.09 & 3.09 \\
\hline 11. & 1.68 & 3.37 & 1.68 & 3.37 & 3.37 & 3.37 & 1.68 \\
\hline 12. & 0.87 & 1.73 & 1.73 & 1.73 & 1.73 & 1.73 & 3.46 \\
\hline 13. & 0.93 & 3.72 & 1.86 & 3.72 & 3.72 & 1.86 & 3.72 \\
\hline DMSO & 0.00 & 0.00 & 0.00 & 0.00 & 0.00 & 0.00 & 0.00 \\
\hline Cefadroxil & 1.72 & 1.72 & 1.72 & 1.72 & 1.72 & - & - \\
\hline Fluconazole & - & - & - & - & - & 2.04 & 2.04 \\
\hline
\end{tabular}

MIC determination into fresh medium. The $\mathrm{MBC}$ and MFC values represent the lowest concentration of compound that produces in the range of $96-98 \%$ end point reduction of the used test bacterial and fungal species [19].

\section{SAR (structure activity relationship) studies}

From the antimicrobial testing results of synthesized 4-(substituted phenyl)-6-(4-nitrophenyl)pyrimidin-2-ol/ thiol/amine derivatives, the subsequent structure activity relationship can be derived in Fig. 2. 
- Presence of electron withdrawing group $(-\mathrm{Cl}$, Compounds 2, 5 and 12) on benzylidene portion improved the antimicrobial activity of the synthesized compounds against $S$. aureus, E. coli, B. subtilis and C. albicans.

- Presence of electron withdrawing group ( $-\mathrm{Br}$, Compound 11) improved the antifungal activity of the synthesized compounds against $A$. niger.

- Using 5-bromo-2-hydroxybenzaldehyde (Compound 10) improved the antibacterial activity of the synthesized compounds against Gram negative S. enterica and $P$. aeruginosa.

- $\mathrm{NO}_{2}$ group presence on benzylidene portion of acetophenone play an important role to enhanced the antimicrobial activity against bacterial and fungal microorganism.

\section{Experimental section}

Starting materials were obtained from commercial sources and were used without any type of further purification. The completion of the chemical reaction was observed by thin layer chromatography (TLC) making use of silica gel $\mathrm{G}$ plates of $0.5 \mathrm{~mm}$ thickness as stationary phase and benzene as mobile phase for final compounds. Melting points of final compounds were determined by open capillary tubes method. The molecular structures of the compounds were characterized by ${ }^{1} \mathrm{H} /{ }^{13} \mathrm{C}-\mathrm{NMR}$ $\left(\mathrm{CDCl}_{3}, \delta \mathrm{ppm}\right), \mathrm{FT}-\mathrm{IR}$ and Mass spectral studies. The Mass spectral data were confirmed by Waters Micromass Q-ToF Micro instrument. ${ }^{1} \mathrm{H}$ nuclear magnetic resonance $\left({ }^{1} \mathrm{H}-\mathrm{NMR}\right)$ spectra was recorded on Bruker Avance
$400 \mathrm{MHz}$ spectrometer in appropriate $\mathrm{CDCl}_{3}$ solvents and are expressed in parts per million $(\delta, \mathrm{ppm})$ downfield from tetramethyl silane (internal standard). ${ }^{1} \mathrm{H}$ NMR data are given as multiplicity (s, singlet; d, doublet; $\mathrm{t}$, triplet; $\mathrm{m}$, multiplet) and number of protons. Infrared (IR) spectra were recorded on Bruker 12060280, Software: OPUS 7.2.139.1294 spectrometer in the range of $400-4000$ using $\mathrm{KBr}$ pellets and the value of $\lambda$ max were reported in $\mathrm{cm}^{-1}$.

\section{General procedure for synthesized pyrimidine analogues}

Step $i$ : synthesis of substituted chalcone (intermediate-I) The reaction mixture of 1-(4-nitrophenyl)ethanone $(0.01 \mathrm{~mol})$ and corresponding aldehyde $(0.01 \mathrm{~mol})$ were stirred for $2-3 \mathrm{~h}$ in methanol $(5-10 \mathrm{ml})$ followed by drop wise addition of sodium hydroxide solution $(10 \mathrm{ml}$ $40 \%$ ) with constant stirring at room temperature. Then reaction mixture was taken overnight at room temperature and then was poured into ice cold water and acidified with hydrochloric acid and the precipitated substituted chalcone was filtered, dried and recrystallized from methanol [20].

Step ii: synthesis of 4-(substituted phenyl)-6-(4-nitrophenyl)pyrimidin-2-ol/thiol/amine derivatives The solution of substituted chalcone $(0.01 \mathrm{~mol})$ [synthesized in "Step i: synthesis of substituted chalcone (intermediate-I)"] in methanol $(50 \mathrm{ml})$ was added with $0.01 \mathrm{~mol}$ of potassium hydroxide and $40 \mathrm{ml}$ of $0.25 \mathrm{M}$ solution of thiourea/urea/ guanidine hydrochloride and refluxed for 3-4 $\mathrm{h}$. The reaction mixture was then cooled and acidified with few drops

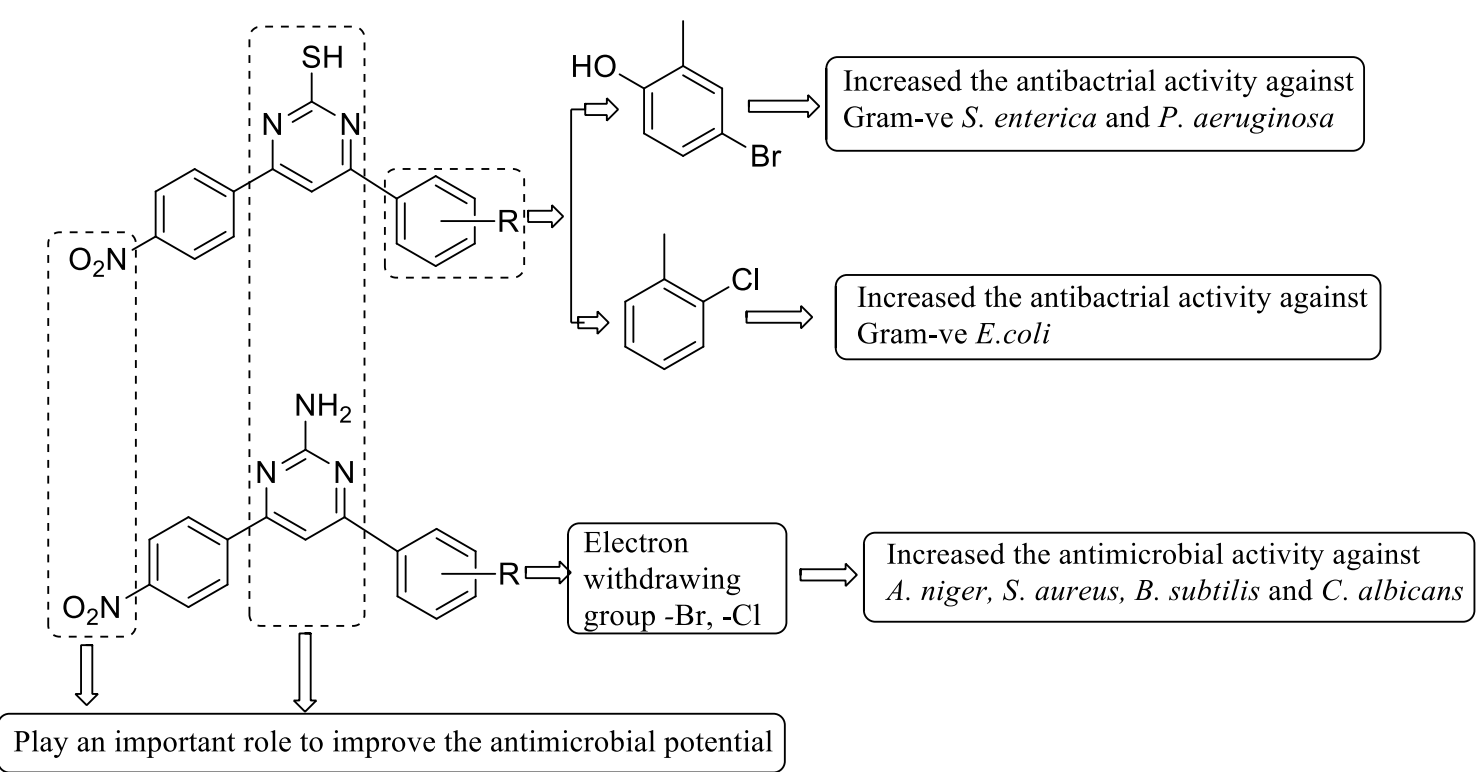

Fig. 2 Structural requirements for the antimicrobial activity of the synthesized derivatives 
of hydrochloric acid ( $20 \mathrm{ml}$ of $0.5 \mathrm{M}$ solution) and the resultant precipitate 4-(substituted phenyl)-6-(4-nitrophenyl)pyrimidin-2-ol/thiol/amine was separated dried and recrystallized from methanol.

Spectral analysis determined by FT-IR (KBr pellets, $\left.\mathrm{cm}^{-1}\right)$ and ${ }^{1} \mathrm{H} /{ }^{13} \mathrm{C}-\mathrm{NMR}\left(\mathrm{CDCl}_{3}, \delta \mathrm{ppm}\right)$.

4-(2-Chlorophenyl)-6-(4-nitrophenyl)pyrimidin-2-amine (1) M. Formula: $\mathrm{C}_{16} \mathrm{H}_{11} \mathrm{ClN}_{4} \mathrm{O}_{2}$; Yield: $75.00 \%$; $\mathrm{MS}$ $\mathrm{ES}+(\mathrm{ToF}): m / z 326\left[\mathrm{M}^{+}+1\right] ; \mathrm{IR}\left(\mathrm{KBr}\right.$ pellets, $\left.\mathrm{cm}^{-1}\right): 2931$ (C-H str.), 1596 ( $\mathrm{C}=\mathrm{C}$ str.), 700 (C-C str.), 1688 ( $\mathrm{C}=\mathrm{N}$ str. or $\mathrm{N}=\mathrm{CH}$ str., pyrimidine ring), $1344(\mathrm{C}-\mathrm{N}$ str., pyrimidine), 754 (C-Cl str.), 1521 ( $\mathrm{NO}_{2}$ asym str.), 854 (C-N str., $\left.\mathrm{Ar}-\mathrm{NO}_{2}\right), 3379\left(\mathrm{NH}_{2}\right.$ asym str.); ${ }^{13} \mathrm{C}-\mathrm{NMR}\left(\mathrm{CDCl}_{3}-d_{6}, \delta\right.$, ppm): 163.4, 163.6, 160.1, 148.3, 139.8, 132.4, 130.1, 129.2, 128.3, 127.4, 121.7, 95.2; ${ }^{1} \mathrm{H}-\mathrm{NMR}\left(\mathrm{CDCl}_{3}, \delta\right.$, ppm): 7.13$8.25(\mathrm{~m}, 8 \mathrm{H}, \mathrm{Ar}-\mathrm{H}), 6.71(\mathrm{~s}, 1 \mathrm{H}, \mathrm{CH}$ of pyrimidine ring), $4.2\left(\mathrm{~s}, 2 \mathrm{H}, \mathrm{NH}_{2}\right)$.

4-(2-Chlorophenyl)-6-(4-nitrophenyl)pyrimidine-2-thiol (2) M. Formula: $\mathrm{C}_{16} \mathrm{H}_{10} \mathrm{ClN}_{3} \mathrm{O}_{2} \mathrm{~S}$; Yield: $84.72 \%$; $\mathrm{MS}$ $\mathrm{ES}+(\mathrm{ToF}): \mathrm{m} / z 343\left[\mathrm{M}^{+}+1\right] ; \mathrm{IR}\left(\mathrm{KBr}\right.$ pellets, $\left.\mathrm{cm}^{-1}\right): 2858$ (C-H str.), 1596 ( $\mathrm{C}=\mathrm{C}$ str.), 703 (C-C str.), 1665 ( $\mathrm{C}=\mathrm{N}$ str.), 1342 (C-N str., pyrimidine), 753 (C-Cl str.), 1521 ( $\mathrm{NO}_{2}$ asym str.), 698 (C-N str., $\mathrm{Ar}-\mathrm{NO}_{2}$ ), 2627 (S-H str.), 621 (C-S str.); ${ }^{13} \mathrm{C}-\mathrm{NMR}\left(\mathrm{CDCl}_{3}-d_{6}, \delta, \mathrm{ppm}\right): 182.4,163.5$, 163.2, 160.1, 147.3, 139.6, 132.2, 130.1, 129.6, 128.3, 127.4, $121.7,106.1 ;{ }^{1} \mathrm{H}-\mathrm{NMR}\left(\mathrm{CDCl}_{3}, \delta, \mathrm{ppm}\right): 7.35-8.34(\mathrm{~m}, 8 \mathrm{H}$, $\mathrm{Ar}-\mathrm{H}), 8.40(\mathrm{~s}, 1 \mathrm{H}, \mathrm{CH}$ of pyrimidine ring), $3.01(\mathrm{~s}, 1 \mathrm{H}, \mathrm{SH})$.

4-(3-Chlorophenyl)-6-(4-nitrophenyl)pyrimidin-2-amine (3) M. Formula: $\mathrm{C}_{16} \mathrm{H}_{11} \mathrm{ClN}_{4} \mathrm{O}_{2}$; Yield: $78.78 \%$; $\mathrm{MS}$ $\mathrm{ES}+(\mathrm{ToF}): m / z 326\left[\mathrm{M}^{+}+1\right]$; IR ( $\mathrm{KBr}$ pellets, $\left.\mathrm{cm}^{-1}\right)$ : 2923 (C-H str.), 1607 (C=C str.), 703 (C-C str.), 1670 (C=N str.), 1351 (C-N str., pyrimidine), 732 (C-Cl str.), $1525\left(\mathrm{NO}_{2}\right.$ asym str., phenyl ring), $674(\mathrm{C}-\mathrm{N}$ str., $\mathrm{Ar}-$ $\left.\mathrm{NO}_{2}\right), 3387\left(\mathrm{NH}_{2}\right.$ asym str.); ${ }^{13} \mathrm{C}-\mathrm{NMR}\left(\mathrm{CDCl}_{3}-d_{6}, \delta\right.$, ppm): 163.2, 160.1, 147.2, 138.6, 132.0, 134.3, 130.1, 129.2, 128.1, 127.4, 125.3, 121.7, 95.3; ${ }^{1} \mathrm{H}-\mathrm{NMR}\left(\mathrm{CDCl}_{3}, \delta, \mathrm{ppm}\right)$ : 7.26-9.02 (m, 8H, Ar-H), $6.0(\mathrm{~s}, 1 \mathrm{H}, \mathrm{CH}$ of pyrimidine ring), $4.3\left(\mathrm{~s}, 2 \mathrm{H}, \mathrm{NH}_{2}\right)$.

4-(3-Chlorophenyl)-6-(4-nitrophenyl)pyrimidine-2-thiol (4) M. Formula: $\mathrm{C}_{16} \mathrm{H}_{10} \mathrm{ClN}_{3} \mathrm{O}_{2} \mathrm{~S}$; Yield: $72.54 \%$; MS $\mathrm{ES}+(\mathrm{ToF}): m / z 343\left[\mathrm{M}^{+}+1\right]$; IR ( $\mathrm{KBr}$ pellets, $\left.\mathrm{cm}^{-1}\right)$ : 2991 (C-H str.), 1570 (C=C str.), 709 (C-C str.), 1701 $(\mathrm{C}=\mathrm{N}$ str. pyrimidine ring), $1303(\mathrm{C}-\mathrm{N}$ str.), $748(\mathrm{C}-\mathrm{Cl}$ str.), 1521 ( $\mathrm{NO}_{2}$ asym str.), 659 (C-N str., $\left.\mathrm{Ar}-\mathrm{NO}_{2}\right), 2597$ (S-H str.), 709 (C-S str.); ${ }^{13} \mathrm{C}-\mathrm{NMR}\left(\mathrm{CDCl}_{3}-d_{6}, \delta, \mathrm{ppm}\right)$ : $181.4,163.5,163.2,160.1,146.3, \quad 139.6, \quad 132.2,130.1,129.6$, 128.3,127.4, 125.3, 121.7, 103.1; ${ }^{1} \mathrm{H}-\mathrm{NMR}\left(\mathrm{CDCl}_{3}, \delta\right.$, ppm): $7.83-8.25(\mathrm{~m}, 8 \mathrm{H}, \mathrm{Ar}-\mathrm{H}), 7.41(\mathrm{~s}, 1 \mathrm{H}, \mathrm{CH}$ of pyrimidine ring), $3.06(\mathrm{~s}, 1 \mathrm{H}, \mathrm{SH})$.
4-(4-Chlorophenyl)-6-(4-nitrophenyl)pyrimidin-2-amine (5) M. Formula: $\mathrm{C}_{16} \mathrm{H}_{11} \mathrm{ClN}_{4} \mathrm{O}_{2}$; Yield: $82.22 \%$; $\mathrm{MS}$ $\mathrm{ES}+(\mathrm{ToF}): m / z 326\left[\mathrm{M}^{+}+1\right]$; IR ( $\mathrm{KBr}$ pellets, $\left.\mathrm{cm}^{-1}\right)$ : 2942 (C-H str.), 1598 (C=C str.), 703 (C-C str.), 1673 ( $\mathrm{C}=\mathrm{N}$ str.), 1346 (C-N str., pyrimidine), 755 (C-Cl str.), $1523\left(\mathrm{NO}_{2}\right.$ asym str.), 822 (C-N str., $\left.\mathrm{Ar}-\mathrm{NO}_{2}\right), 3349\left(\mathrm{NH}_{2}\right.$ asym str.); ${ }^{13} \mathrm{C}-\mathrm{NMR}\left(\mathrm{CDCl}_{3}-d_{6}, \delta, \mathrm{ppm}\right): 162.2,160.1$, 146.2, 138.6, 131.0, 134.3 130.1, 129.2, 128.1, 127.4, 124.3, 121.7, 96.3; ${ }^{1} \mathrm{H}-\mathrm{NMR}\left(\mathrm{CDCl}_{3}, \delta, \mathrm{ppm}\right): 7.33-8.34(\mathrm{~m}, 8 \mathrm{H}$, $\mathrm{Ar}-\mathrm{H}), 7.85$ (s, $1 \mathrm{H}, \mathrm{CH}$ of pyrimidine ring), $4.14(\mathrm{~s}, 2 \mathrm{H}$, $\mathrm{NH}_{2}$ ).

4-(4-Chlorophenyl)-6-(4-nitrophenyl)pyrimidine-2-thiol (6) M. Formula: $\mathrm{C}_{16} \mathrm{H}_{10} \mathrm{ClN}_{3} \mathrm{O}_{2} \mathrm{~S}$; Yield: $75.00 \%$; $\mathrm{MS}$ $\mathrm{ES}+(\mathrm{ToF}): m / z 343\left[\mathrm{M}^{+}+1\right] ; \mathrm{IR}\left(\mathrm{KBr}\right.$ pellets, $\left.\mathrm{cm}^{-1}\right): 2927$ (C-H str.), 1596 (C=C str.), 709 (C-C str.), 1345 (C-N str., pyrimidine), 824 (C-Cl str.), $1480\left(\mathrm{NO}_{2}\right.$ asym str.), 698 (C-N str., Ar- $\mathrm{NO}_{2}$ ), 645 (C-S str.), 2602 (S-H str.); ${ }^{13} \mathrm{C}-\mathrm{NMR}\left(\mathrm{CDCl}_{3}-d_{6}, \delta\right.$, ppm): 182.4, 163.2, 163.2, 161.1, 148.3, 139.6, 131.2, 130.1, 129.6, 128.3, 126.4, 121.7, 103.4; ${ }^{1} \mathrm{H}-\mathrm{NMR}\left(\mathrm{CDCl}_{3}, \delta, \mathrm{ppm}\right): 7.83-8.25(\mathrm{~m}, 8 \mathrm{H}, \mathrm{Ar}-\mathrm{H}), 7.45$ (s, $1 \mathrm{H}, \mathrm{CH}$ of pyrimidine ring), $3.34(\mathrm{~s}, 1 \mathrm{H}, \mathrm{SH})$.

4-(4-Chlorophenyl)-6-(4-nitrophenyl)pyrimidin-2-ol (7) M. Formula: $\mathrm{C}_{16} \mathrm{H}_{10} \mathrm{ClN}_{3} \mathrm{O}_{3}$; Yield: $84.72 \%$; $\mathrm{MS}$ $\mathrm{ES}+(\mathrm{ToF}): m / z 327\left[\mathrm{M}^{+}+1\right]$; IR (KBr pellets, $\left.\mathrm{cm}^{-1}\right)$ : 2941 (C-H str.), 1595 (C=C str.), 705 (C-C str.), 1672 (C=N str.), 1342 (C-N str., pyrimidine), 756 (C-Cl str.), 1523 ( $\mathrm{NO}_{2}$ asym str.), 3374 (O-H str.), 822 (C-N str., Ar$\left.\mathrm{NO}_{2}\right), 1404(\mathrm{C}-\mathrm{O}$ str., and $\mathrm{O}-\mathrm{H}$ in plane bending vib.); ${ }^{13} \mathrm{C}$-NMR $\left(\mathrm{CDCl}_{3}-d_{6}, \delta\right.$, ppm): 160.4, 160.4, 153.2, 148.2, 139.1, 134.1, 131.1, 129.2, 128.2, 121.2, 88.1; ${ }^{1} \mathrm{H}-\mathrm{NMR}$ $\left(\mathrm{CDCl}_{3}, \delta, \mathrm{ppm}\right): 7.43-8.56(\mathrm{~m}, 8 \mathrm{H}, \mathrm{Ar}-\mathrm{H}), 6.61(\mathrm{~s}, 1 \mathrm{H}$, $\mathrm{CH}$ of pyrimidine ring), $5.04(\mathrm{~s}, 1 \mathrm{H}, \mathrm{OH})$.

4-(3-Methoxyphenyl)-6-(4-nitrophenyl)pyrimidin-2-amine (8) M. Formula: $\mathrm{C}_{17} \mathrm{H}_{14} \mathrm{~N}_{4} \mathrm{O}_{3}$; Yield: 73.43\%; MS ES + (ToF): $m / z 322\left[\mathrm{M}^{+}+1\right]$; IR (KBr pellets, $\left.\mathrm{cm}^{-1}\right)$ : 2947 (C-H str.), 692 (C-C str.), 1709 ( $\mathrm{C}=\mathrm{N}$ str. pyrimidine ring), 1344 ( $\mathrm{C}-\mathrm{N}$ str., pyrimidine), 784 ( $\mathrm{C}-\mathrm{N}$ str., Ar- $\left.\mathrm{NO}_{2}\right), 1041\left(\mathrm{C}-\mathrm{O}-\mathrm{C}\right.$ str., $\left.-\mathrm{OCH}_{3}\right), 2839(\mathrm{C}-\mathrm{H}$ str., R$\left.\mathrm{CH}_{3}\right) ;{ }^{13} \mathrm{C}-\mathrm{NMR}\left(\mathrm{CDCl}_{3}-d_{6}, \delta, \mathrm{ppm}\right): 163.2,160.1,146.2$, 139.6, 131.0, $134.3130 .1,128.1,121.7,119.3,114.3,111.3$, 96.3, 55.2; ${ }^{1} \mathrm{H}-\mathrm{NMR}\left(\mathrm{CDCl}_{3}, \delta, \mathrm{ppm}\right): 6.33-8.44(\mathrm{~m}, 8 \mathrm{H}$, $\mathrm{Ar}-\mathrm{H}), 6.85(\mathrm{~s}, 1 \mathrm{H}, \mathrm{CH}$ of pyrimidine ring), $4.2(\mathrm{~s}, 2 \mathrm{H}$, $\left.\mathrm{NH}_{2}\right), 3.34\left(\mathrm{~s}, 1 \mathrm{H}, \mathrm{OCH}_{3}\right)$.

4-(4-Methoxyphenyl)-6-(4-nitrophenyl)pyrimidin-2-amine (9) M. Formula: $\mathrm{C}_{17} \mathrm{H}_{14} \mathrm{~N}_{4} \mathrm{O}_{3}$; Yield: 76.47\%; MS ES + (ToF): $m / z 322\left[\mathrm{M}^{+}+1\right]$; IR (KBr pellets, $\mathrm{cm}^{-1}$ ): 2937 (C-H str.), 1604 (C=C str.), 694 (C-C str.), 1661 (C=N str.), 1349 (C-N str., pyrimidine), 1502 $\left(\mathrm{NO}_{2}\right.$ asym str., phenyl ring), $752\left(\mathrm{C}-\mathrm{N}\right.$ str., $\left.\mathrm{Ar}-\mathrm{NO}_{2}\right)$, 
$1108\left(\mathrm{C}-\mathrm{O}-\mathrm{C}\right.$ str., $\left.-\mathrm{OCH}_{3}\right), 2842\left(\mathrm{C}-\mathrm{H}\right.$ str., $\left.\mathrm{R}-\mathrm{CH}_{3}\right)$; ${ }^{13} \mathrm{C}-\mathrm{NMR}\left(\mathrm{CDCl}_{3}-d_{6}, \delta, \mathrm{ppm}\right): 163.1,160.1,148.2,139.6$, 128.1, 125.3, 121.7, 114.3, 95.3; ${ }^{1} \mathrm{H}-\mathrm{NMR}\left(\mathrm{CDCl}_{3}, \delta, \mathrm{ppm}\right)$ : $6.33-8.71(\mathrm{~m}, 8 \mathrm{H}, \mathrm{Ar}-\mathrm{H}), 6.35(\mathrm{~s}, 1 \mathrm{H}, \mathrm{CH}$ of pyrimidine ring), $4.23\left(\mathrm{~s}, 2 \mathrm{H}, \mathrm{NH}_{2}\right), 3.01\left(\mathrm{~s}, 1 \mathrm{H}, \mathrm{OCH}_{3}\right)$.

4-Bromo-2-(2-mercapto-6-(4-nitrophenyl)pyrimidin-4-yl)phenol (10) M. Formula: $\mathrm{C}_{16} \mathrm{H}_{10} \mathrm{BrN}_{3} \mathrm{O}_{3} \mathrm{~S}$; Yield: 81.81\%; MS ES + (ToF): $m / z 404\left[\mathrm{M}^{+}+1\right]$; IR ( $\mathrm{KBr}$ pellets, $\mathrm{cm}^{-1}$ ): 2869 (C-H str.), $1592(\mathrm{C}=\mathrm{C}$ str.), 691 (C-C str.), 1680 (C=N str.), 1349 (C-N str., pyrimidine), 623 (C-Br str.), 1521 ( $\mathrm{NO}_{2}$ asym str., phenyl ring), 844 (C-N str., Ar- $\mathrm{NO}_{2}$ ), 2597 (S-H str.), 623 (C-S str.); ${ }^{13} \mathrm{C}-\mathrm{NMR}\left(\mathrm{CDCl}_{3}-d_{6}, \delta\right.$, ppm): 182.4, 163.2, 161.1, 154.3, $148.3,139.6,134.2,133.1,128.3,121.2,122.4,115.2$, 118.2, 103.4; ${ }^{1} \mathrm{H}-\mathrm{NMR}\left(\mathrm{CDCl}_{3}, \delta, \mathrm{ppm}\right): 7.93-8.35$ (m, $7 \mathrm{H}, \mathrm{Ar}-\mathrm{H}), 8.41$ (s, $1 \mathrm{H}, \mathrm{CH}$ of pyrimidine ring), 3.05 (s, $1 \mathrm{H}, \mathrm{SH}), 5.97(\mathrm{~s}, 1 \mathrm{H}, \mathrm{OH})$.

4-(3-Bromophenyl)-6-(4-nitrophenyl)pyrimidin-2-amine (11) M. Formula: $\mathrm{C}_{16} \mathrm{H}_{11} \mathrm{BrN}_{4} \mathrm{O}_{2}$; Yield: $64.00 \%$; MS $\mathrm{ES}+(\mathrm{ToF}): m / z 371\left[\mathrm{M}^{+}+1\right]$; IR (KBr pellets, $\left.\mathrm{cm}^{-1}\right)$ : 3064 (C-H str.), 1596 (C=C str.), 692 (C-C str.), 1671 ( $\mathrm{C}=\mathrm{N}$ str.), 1342 (C-N str., pyrimidine), $1500\left(\mathrm{NO}_{2}\right.$ asym str., phenyl ring), 783 (C-N str., $\mathrm{Ar}-\mathrm{NO}_{2}$ ), 645 (C-Br str.); ${ }^{13} \mathrm{C}-\mathrm{NMR}\left(\mathrm{CDCl}_{3}-d_{6}, \delta, \mathrm{ppm}\right): 163.2,160.1,148.2,139.6$, $131.0,134.3,130.1,129.2,128.1,126.3,121.7,95.3 ;{ }^{1} \mathrm{H}-$ NMR $\left(\mathrm{CDCl}_{3}, \delta, \mathrm{ppm}\right): 6.11-8.41(\mathrm{~m}, 8 \mathrm{H}, \mathrm{Ar}-\mathrm{H}), 7.35$ (s, $1 \mathrm{H}, \mathrm{CH}$ of pyrimidine ring $), 4.00\left(\mathrm{~s}, 2 \mathrm{H}, \mathrm{NH}_{2}\right)$.

4-(2,4-Dichlorophenyl)-6-(4-nitrophenyl)pyrimidin-2-amine (12) M. Formula: $\mathrm{C}_{16} \mathrm{H}_{10} \mathrm{ClN}_{4} \mathrm{O}_{2}$; Yield: 87.61\%; MS ES + (ToF): $m / z 361\left[\mathrm{M}^{+}+1\right]$; IR (KBr pellets, $\mathrm{cm}^{-1}$ ):1600 ( $\mathrm{C}=\mathrm{C}$ str.), 695 (C-C str.), $1669(\mathrm{C}=\mathrm{N}$ str.), 1346 (C-N str., pyrimidine), 848 (C-Cl str.), 1415 $\left(\mathrm{NO}_{2}\right.$ asym str., phenyl ring), $735\left(\mathrm{C}-\mathrm{N}\right.$ str., $\left.\mathrm{Ar}-\mathrm{NO}_{2}\right) ;{ }^{1} \mathrm{H}-$ NMR $\left(\mathrm{CDCl}_{3}, \delta, \mathrm{ppm}\right): 6.34-8.67(\mathrm{~m}, 7 \mathrm{H}, \mathrm{Ar}-\mathrm{H}), 6.15$ (s, $1 \mathrm{H}, \mathrm{CH}$ of pyrimidine ring), $4.30\left(\mathrm{~s}, 2 \mathrm{H}, \mathrm{NH}_{2}\right) ;{ }^{13} \mathrm{C}-\mathrm{NMR}$ $\left(\mathrm{CDCl}_{3}-d_{6}, \delta, \mathrm{ppm}\right): 163.6,160.1,147.2,139.4,133.3$, 135.1, 129.2,128.1, 127.3, 121.7, 95.6.

4-(4-(Dimethylamino)phenyl)-6-(4-nitrophenyl)pyrimidin-2-ol (13) M. Formula: $\mathrm{C}_{18} \mathrm{H}_{16} \mathrm{~N}_{4} \mathrm{O}_{3}$; Yield: $77.38 \%$; MS ES + (ToF): $m / z 336\left[\mathrm{M}^{+}+1\right] ;$ IR $\left(\mathrm{KBr}\right.$ pellets, $\left.\mathrm{cm}^{-1}\right)$ : 2923 (C-H str.), 1524 (C=C str.), 704 (C-C str.), 1670 ( $\mathrm{C}=\mathrm{N}$ str. or $\mathrm{N}=\mathrm{CH}$ str., pyrimidine ring), $1348(\mathrm{C}-\mathrm{N}$ str., phenyl ring), $733\left(\mathrm{NO}_{2}\right.$ asym str., phenyl ring), 806 (C-N str., Ar. nitro group), 2858 (C-H str., $\left.\mathrm{R}-\mathrm{CH}_{3}\right), 3393$ (O-H str.); ${ }^{13} \mathrm{C}-\mathrm{NMR}\left(\mathrm{CDCl}_{3}-d_{6}, \delta, \mathrm{ppm}\right): 160.5,154.3$, 149.2, 139.6, 128.1, 122.7,121.3, 114.3, 87.2, 41.1; ${ }^{1} \mathrm{H}-\mathrm{NMR}$ $\left(\mathrm{CDCl}_{3}, \delta, \mathrm{ppm}\right): 6.11-8.26(\mathrm{~m}, 8 \mathrm{H}, \mathrm{Ar}-\mathrm{H}), 6.75(\mathrm{~s}, 1 \mathrm{H}, \mathrm{CH}$ of pyrimidine ring), $5.30(\mathrm{~s}, 1 \mathrm{H}, \mathrm{OH}), 2.19\left(\mathrm{~s}, 6 \mathrm{H},\left(\mathrm{CH}_{3}\right)_{2}\right)$.

\section{Conclusion}

Summarizing, we may conclude that the synthesized compounds $(2,5,10,11$ and 12) displayed appreciable antibacterial and antifungal activities against Gram positive bacteria ( $S$. aureus and B. subtilis), Gram negative bacteria (E. coli, S. enterica and P. aeruginosa) and fungal strains (C. albicans and $A$. niger). The electron withdrawing group's play an important role to enhanced the antimicrobial potential of compounds 2, 5, 11 and 12 and these compound more active than standard drugs cefadroxil and fluconazole. The MBC and MFC values represent the lowest concentration of compound that produces in the range of 96-98\% end point reduction of the used test bacterial and fungal species.

\section{Authors' contributions}

PKV designed and finalized the scheme; SN performed research work and SK analyzed the spectral and biological data and wrote the paper. All authors read and approved the final manuscript.

\section{Acknowledgements}

Thanks to Head, Department of Pharmaceutical Sciences, M. D. University, Rohtak for kind support for providing chemicals etc.

\section{Competing interests}

The authors declare that they have no competing interests.

\section{Publisher's Note}

Springer Nature remains neutral with regard to jurisdictional claims in published maps and institutional affiliations.

Received: 5 April 2017 Accepted: 5 June 2017

Published online: 09 June 2017

\section{References}

1. Sarkar A, Kumar KA, Dutta NK, Chakraborty P, Dastidar SG (2003) Evaluation of in vitro and in vivo antibacterial activity of dobutamine hydrochloride. Indian J Med Microbiol 213:172-178

2. Tomma JH, Khazaal MS, Al-Dujaili AH (2014) Synthesis and characterization of novel Schiff bases containing pyrimidine unit. Arab J Chem 7:157-163

3. Rani J, Kumar S, Saini M, Mundlia J, Verma PK (2016) Biological potential of pyrimidine derivatives in a new era. Res Chem Intermed 42:6777-6804

4. Guo Y, Li Jing, Ma J, Yu Z, Wang H, Zhua J, Liao X, Zhao Y (2015) Synthesis and antitumor activity of $a$-aminophosphonate derivatives containing thieno[2,3-d]pyrimidines. Chin Chem Lett 26:755-758

5. Mallikarjunaswamy C, Mallesha L, Bhadregowda DG, Pinto O (2012) Studies on synthesis of pyrimidine derivatives and their antimicrobial activity. Arab J Chem (in press)

6. Cocco MT, Congiu C, Onnis V, Piras R (2001) Synthesis and antitumor evaluation of 6-thioxo-, 6-oxo- and 2,4-dioxopyrimidine derivatives. II Farmaco 56:741-748

7. Anupama B, Dinda SC, Prasad YR, Rao AV (2012) Synthesis and antimicrobial activity of some new 2,4,6-trisubstituted pyrimidines. Int J Res Pharm Chem 2(2):231-236

8. Tozkoparan B, Ertan M, Kelicen P, Demirdamar R (1999) Synthesis and antiinflammatory activities of some thiazolo[3,2-a]pyrimidine derivatives. II Farmaco 54:588-593

9. Ashour HM, Shaaban OG, Rizk OH, El-Ashmawy IM (2013) Synthesis and biological evaluation of thieno[2', $\left.3^{\prime}: 4,5\right]$ pyrimido[1,2-b][1,2,4]triazines and thieno[2,3-d][1,2,4]triazolo[1,5-a]pyrimidines as anti-inflammatory and analgesic agents. Eur J Med Chem 62:341-351 
10. Bhalgat CM, Ali MI, Ramesh B, Ramu G (2014) Novel pyrimidine and its triazole fused derivatives: synthesis and investigation of antioxidant and anti-inflammatory activity. Arab J Chem 7:986-993

11. Kumar D, Khan SI, Tekwani BL, Diwan PP, Rawat S (2015) 4-Aminoquinoline-pyrimidine hybrids: synthesis, antimalarial activity, heme binding and docking studies. Eur J Med Chem 89:490-502

12. Avupati DVR, Yejella PRP (2014) A review on pyrimidine scaffold. World J Pharm Res Technol 3(10):1563-1587

13. Gupta Y, Gupta V, Singh S (2013) Synthesis, characterization and antimicrobial activity of pyrimidine based derivatives. J Pharm Res 7:491-495

14. Prajapti SK, Nagarsenkar A, Guggilapu SD, Gupta KK, Allakonda L, Jeengar MK, Naidu VG, Babu BN (2016) Synthesis and biological evaluation of oxindole linked indolyl-pyrimidine derivatives as potential cytotoxic agents. Bioorg Med Chem Lett 26(13):3024-3028

15. Bhalgat C, Ramesh B (2014) Synthesis, antimicrobial screening and structure-activity relationship of novel pyrimidines and their thioethers. Bull Fac Pharm Cairo Univ 52:259-267
16. Desai NC, Kotadiya GM, Trivedi AR (2014) Studies on molecular properties prediction, antitubercular and antimicrobial activities of novel quinoline based pyrimidine motifs. Bioorg Med Chem Lett 24:3126-3130

17. Cappuccino JG, Sherman N (1999) In microbiology-a laboratory manual, 4th edn. Addison Wesley Longman Inc, California, p 263

18. Pharmacopoeia of India, vol. I (1985) Controller of Publication, Ministry of Health Department, Govt. of India, New Delhi, p 37

19. Sharma D, Narasimhan B, Kumar $P$, Judge V, Narang R, Clercq ED, Balzarini J (2009) Synthesis, antimicrobial and antiviral activity of substituted benzimidazoles. J Enzyme Inhib Med Chem 24(5):1161-1168

20. Kachroo M, Panda R, Yadav Y (2014) Synthesis and biological activities of some new pyrimidine derivatives from chalcones. Pharm Chem 6(2):352

\section{Submit your manuscript to a SpringerOpen ${ }^{\odot}$ journal and benefit from:}

- Convenient online submission

- Rigorous peer review

- Immediate publication on acceptance

- Open access: articles freely available online

- High visibility within the field

- Retaining the copyright to your article 